\title{
Improved $p$-hydroxybenzoate production by engineered Pseudomonas putida S12 by using a mixed-substrate feeding strategy
}

\author{
Jean-Paul Meijnen • Suzanne Verhoef • \\ Ashwin A. Briedjlal • Johannes H. de Winde • \\ Harald J. Ruijssenaars
}

Received: 6 October 2010 /Revised: 18 December 2010 /Accepted: 31 December 2010 /Published online: 2 February 2011

(C) The Author(s) 2011. This article is published with open access at Springerlink.com

\begin{abstract}
The key precursors for $p$-hydroxybenzoate production by engineered Pseudomonas putida S12 are phosphoenolpyruvate (PEP) and erythrose-4-phosphate (E4P), for which the pentose phosphate (PP) pathway is an important source. Since PP pathway fluxes are typically low
\end{abstract}

Jean-Paul Meijnen and Suzanne Verhoef contributed equally to this work.

J.-P. Meijnen · S. Verhoef • A. A. Briedjlal · J. H. de Winde $(\triangle)$ Department of Biotechnology, Delft University of Technology, Julianalaan 67 ,

2628 BC Delft, The Netherlands

e-mail: J.H.deWinde@tudelft.nl

J.-P. Meijnen $\cdot$ S. Verhoef $\cdot$ J. H. de Winde $\cdot$ H. J. Ruijssenaars B-Basic,

Julianalaan 67 ,

2628 BC Delft, The Netherlands

J.-P. Meijnen $\cdot$ S. Verhoef $\cdot$ J. H. de Winde $\cdot$ H. J. Ruijssenaars Kluyver Centre for Genomics of Industrial Fermentation, P.O. Box 5057, 2600 GA Delft, The Netherlands

\section{H. J. Ruijssenaars}

BIRD Engineering BV,

Westfrankelandsedijk 1,

3115 HG Schiedam, The Netherlands

Present Address:

J.-P. Meijnen

Laboratory of Molecular Cell Biology, Institute of Botany

and Microbiology, Katholieke Universiteit Leuven,

Kasteelpark Arenberg 31,

B-3001 Leuven-Heverlee, Flanders, Belgium

Present Address:

S. Verhoef

Purac, Arkelsedijk 46,

4206 AC Gorinchem, The Netherlands in pseudomonads, E4P and PEP availability is a likely bottleneck for aromatics production which may be alleviated by stimulating PP pathway fluxes via co-feeding of pentoses in addition to glucose or glycerol. As P. putida S12 lacks the natural ability to utilize xylose, the xylose isomerase pathway from $E$. coli was introduced into the $p$-hydroxybenzoate producing strain P. putida S12palB2. The initially inefficient xylose utilization was improved by evolutionary selection after which the $p$-hydroxybenzoate production was evaluated. Even without xylose-co-feeding, $p$-hydroxybenzoate production was improved in the evolved xyloseutilizing strain, which may indicate an intrinsically elevated PP pathway activity. Xylose co-feeding further improved the $p$-hydroxybenzoate yield when co-fed with either glucose or glycerol, up to $16.3 \mathrm{Cmol} \%$ (0.1 g $p$-hydroxybenzoate/g substrate). The yield improvements were most pronounced with glycerol, which probably related to the availability of the PEP precursor glyceraldehyde-3-phosphate (GAP). Thus, it was demonstrated that the production of aromatics such as $p$-hydroxybenzoate can be improved by co-feeding different carbon sources via different and partially artificial pathways. Moreover, this approach opens new perspectives for the efficient production of (fine) chemicals from renewable feedstocks such as lignocellulose that typically has a high content of both glucose and xylose and (crude) glycerol.

Keywords Pseudomonas putida S12 - Aromatics · Mixed substrate feeding $\cdot$ Glucose $\cdot$ Xylose

\section{Introduction}

Pseudomonas putida $\mathrm{S} 12$ is a solvent-tolerant bacterium that has been developed as a platform host for the production of 
a range of substituted aromatic compounds such as phenol, $t$ cinnamate, $p$-coumarate, $p$-hydroxybenzoate, and $p$-hydroxystyrene (Nijkamp et al. 2005; Nijkamp et al. 2007; Verhoef et al. 2007; Verhoef et al. 2009; Wierckx et al. 2005). Its solvent tolerance properties enable $P$. putida $\mathrm{S} 12$ to produce these toxic hydrophobic compounds to high titres without provoking harmful effects (de Bont 1998). Furthermore, in situ product extraction can be applied in fermentations by adding a second phase of a water-immiscible solvent, preventing the accumulation of product to concentrations that are inhibitory even to solvent-tolerant microorganisms (Heipieper et al. 2007; Verhoef et al. 2009).

The production of aromatic compounds by engineered $P$. putida $\mathrm{S} 12$ is based on the conversion of endogenously formed tyrosine or phenylalanine. The key precursors of these aromatic amino acids are phosphoenolpyruvate (PEP) and erythrose-4-phosphate (E4P) (Fig. 1). PEP is produced in the lower glycolysis from glyceraldehyde-3-phosphate (GAP). GAP is formed from glucose, either via the EntnerDoudoroff pathway or via the pentose phosphate (PP) pathway, whereas E4P is derived exclusively from the PP pathway. In view of the typically low activity of the PP pathway in P. putida (del Castillo et al. 2007; Fuhrer et al.
2005; Wierckx et al. 2009), the availability of E4P and PEP may present a bottleneck for efficient aromatics production. Increasing the availability of E4P and PEP was therefore expected to enhance the production of aromatic compounds by engineered $P$. putida $\mathrm{S} 12$, as previously demonstrated for the pre-aromatic compounds chorismate and shikimate in Escherichia coli (Martinez et al. 2008).

The availability of PEP and E4P may be improved by stimulating PP pathway fluxes through pentose (co-) feeding, as was demonstrated previously in $E$. coli (Gonzalez et al. 2002). Unfortunately, this strategy cannot be applied to $P$. putida S12 as this strain lacks the natural ability to utilize pentoses. However, in previous work, we successfully introduced xylose utilization, via the xylose isomerase and PP pathway, into wild-type P. putida $\mathrm{S} 12$ (Meijnen et al. 2008). In the present study, a similar approach was employed to introduce xylose catabolism into $P$. putida S12palB2. This $P$. putida $\mathrm{S} 12$-derived strain produces $p$-hydroxybenzoate, which was selected as a model value-added aromatic compound derived from the aromatic amino acid biosynthesis pathway (Verhoef et al. 2010). The effect of xylose co-feeding on $p$-hydroxybenzoate production was assessed using glucose as the primary
Fig. 1 Schematic representation of the biosynthetic pathways for $p$ hydroxybenzoate production from glycerol, glucose, and xylose. The scheme shows only the relevant routes. Heterologous genes are indicated in italics and underlined. Xylose isomerase (xylA); xylulokinase $(x y l B)$; phenylalanine/tyrosine ammonia lyase (pal/ $t a l)$. Glucose-6-phosphate $(G 6 P)$; fructose-6-phosphate $(F 6 P)$; fructose-1,6-bisphosphate $(F 1,6 B P)$; triose-3-phosphate (T3P); phosphoenolpyruvate $(P E P)$; pyruvate $(P Y R)$; glycerol3-phosphate $(G l y 3 P)$; ribulose-5phosphate (RU5P); xylulose-5phosphate $(X u 5 P)$; ribose-5phosphate $(R 5 P)$; glyceraldehyde3-phosphate $(G A P)$; sedoheptulose-7-phosphate $(S 7 P)$; erythrose-4-phosphate $(E 4 P)$; 3deoxy-D-arabino-heptulosonate-7phosphate $(D A H P)$; chorismate $(\mathrm{CHO})$; phenylalanine $(\mathrm{PHE})$; cinnamate $(C I N)$; tyrosine $(T Y R)$; p-coumarate (COUM); 4hydroxyphenylpyruvate degradation pathway (HD pathway); protocatechuate degradation pathway (PD Pathway)

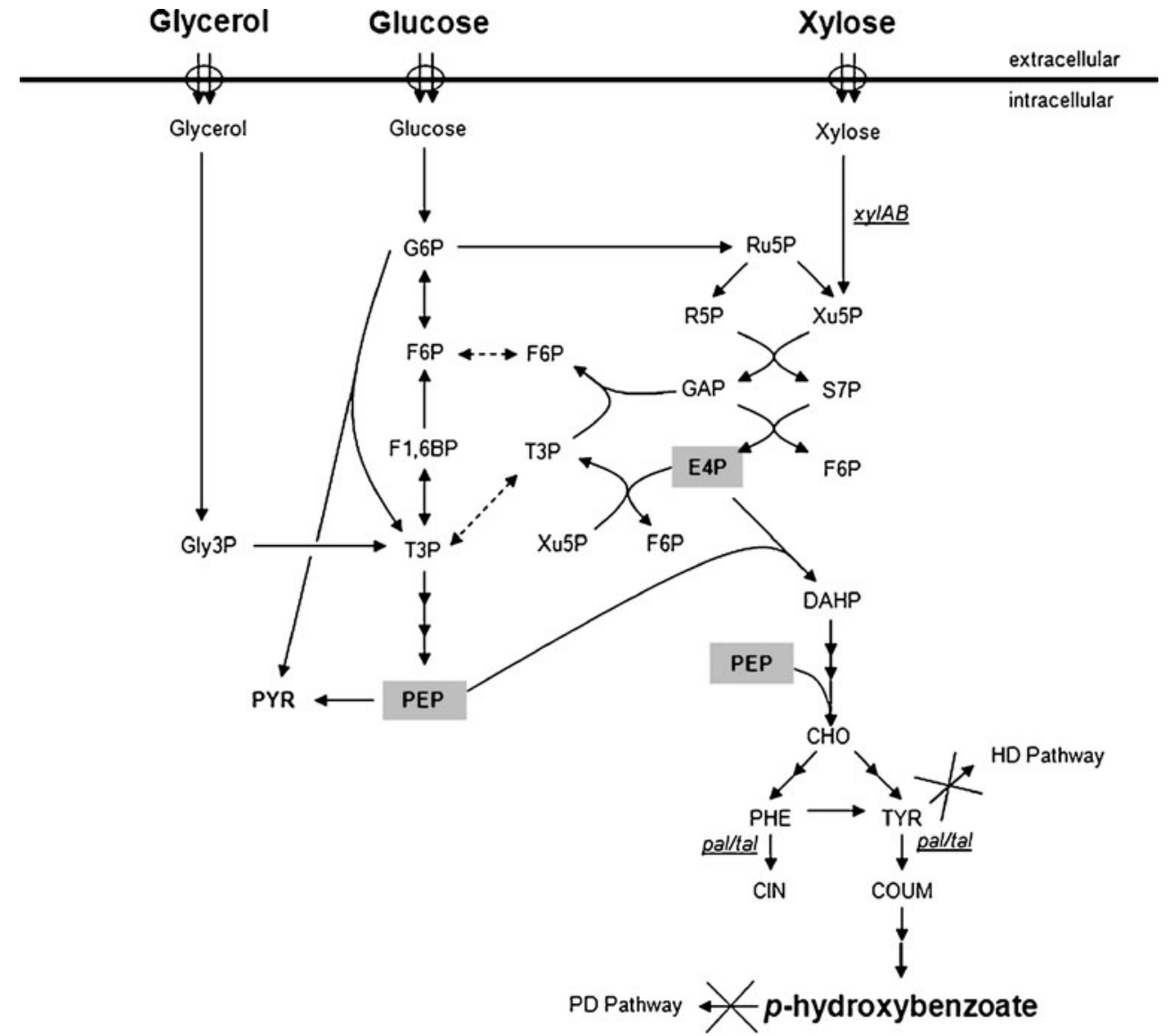


carbon source, mimicking lignocellulosic hydrolysate that typically contains high levels of both glucose and xylose. Alternatively, glycerol was employed as primary carbon source, being a good source for PEP as well as being a model for raw glycerol waste from biodiesel production.

\section{Materials and methods}

Bacterial strains, plasmids, and culture conditions

The strains and plasmids used in this study are listed in Table 1. The media used were Luria broth (LB) (Sambrook et al. 1982) and a phosphate-buffered minimal medium (MM; (Verhoef et al. 2010)). In minimal media, $12 \mathrm{mM}$ of xylose (MMX), $10 \mathrm{mM}$ of glucose (MMG), or $20 \mathrm{mM}$ of glycerol (MMGly) was used as sole carbon source, unless stated otherwise. Antibiotics were added as required to the media to the following final concentrations: gentamicin, $10 \mu \mathrm{g} \mathrm{ml}$ (MM) or $25 \mu \mathrm{g} \mathrm{m} \mathrm{m}^{-1}$ (LB); tetracycline, $10 \mu \mathrm{g} \mathrm{ml}^{-1}$ (E. coli), $40 \mu \mathrm{g} \mathrm{ml}^{-1}$ (P. putida $\mathrm{S} 12$ in MM) or $60 \mu \mathrm{g} \mathrm{ml}^{-1}$ (P. putida $\mathrm{S} 12$ in LB). In view of the photosensitivity of tetracycline, amber bottles and light tight fermentors were employed for culturing. The expression of an additional copy of the native aroF-1 gene (Wierckx et al. 2005) as well as the expression of the $x y l A B \_F G H$ genes from $E$. coli (both under the control of the nagAa promoter) were induced by addition of $0.1 \mathrm{mM}$ of sodium salicylate.

Batch experiments were performed in boston bottles containing $20 \mathrm{ml}$ of minimal medium in a horizontally shaking incubator at $30^{\circ} \mathrm{C}$. Cultures were inoculated to a starting optical density at $600 \mathrm{~nm}\left(\mathrm{OD}_{600}\right)$ of 0.1 with cells precultured on MM with xylose, glucose, or glycerol, depending on the carbon source to be used.

Carbon-limited chemostat cultivations were performed as described previously (Verhoef et al. 2007) in 11 fermentors with a BioFlo110 controller (New Brunswick Scientific) using MM containing different mixtures of glycerol and xylose or glucose and xylose (total carbon (C) concentration, $60 \mathrm{C} \mathrm{mM}), 10 \mu \mathrm{g} \mathrm{ml} \mathrm{m}^{-1}$ gentamicin, $40 \mu \mathrm{g} \mathrm{ml}{ }^{-1}$ tetracycline, and $0.1 \mathrm{mM}$ sodium salicylate. Chemostats were inoculated with a $35-\mathrm{ml}$ inoculum of a late-log phase preculture on MMX. For chemostat cultivations on xylose as sole carbon source, the dilution rate (D) was set at $0.05 \mathrm{~h}^{-1}$ since wash-out occurred at higher dilution rates. For glycerol-xylose mixtures, the $D$ was initially set to $0.05 \mathrm{~h}^{-1}$. When steady state was reached, the cultures were sampled and $D$ was increased to $0.1 \mathrm{~h}^{-1}$.

Table 1 Strains and plasmids used in this study

\begin{tabular}{|c|c|c|}
\hline Strain or plasmid & Characteristics $^{\mathrm{a}}$ & Source \\
\hline \multicolumn{3}{|l|}{ Strains } \\
\hline P. putida $\mathrm{S} 12$ & Wild type, ATCC 700801 & (Hartmans et al. 1990) \\
\hline P. putida $\mathrm{S} 12 \mathrm{~B} 2$ & pobA and $h p d$ knockout strain with an enhanced flux towards tyrosine & (Verhoef et al. 2010) \\
\hline P. putida $\mathrm{S} 12 \mathrm{palB} 2$ & P. putida $\mathrm{S} 12 \mathrm{~B} 2$ containing plasmid $\mathrm{pJT}$ 'Tpal & (Verhoef et al. 2010) \\
\hline P. putida $\mathrm{S} 12 \mathrm{~B} 6$ & gcd knockout strain derived from $P$. putida $\mathrm{S} 12 \mathrm{~B} 2$ & This study \\
\hline P. putida S12palB6 & P. putida $\mathrm{S} 12 \mathrm{~B} 6$ containing plasmid $\mathrm{pBT}$ 'Tpal & This study \\
\hline P. putida $\mathrm{S} 12 \mathrm{xylB} 6$ & P. putida S12B6 containing plasmid pJNTxylAB_FGH & This study \\
\hline P. putida $\mathrm{S} 12 \mathrm{xylB} 7$ & P. putida S12xylB6 evolved to efficient xylose utilizer & This study \\
\hline P. putida S12pal_xylB7 & P. putida $\mathrm{S} 12 \mathrm{xy} 1 \mathrm{~B} 7$ containing plasmid $\mathrm{pBT}$ 'Tpal & This study \\
\hline Escherichia coli $\mathrm{DH} 5 \alpha$ & $\begin{array}{l}\text { supE44 } \Delta \text { lac } \mathrm{U} 169(\phi 80 \text { lacZ } \Delta \mathrm{M} 15) \text { hsdR17 recA1 } \\
\text { endA1 gyrA96 thi-1 relA1 }\end{array}$ & Invitrogen \\
\hline \multicolumn{3}{|c|}{ 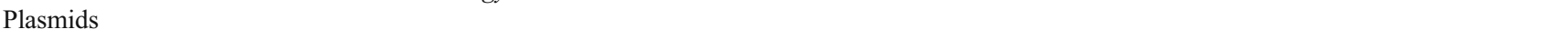 } \\
\hline pJT'Tmcs & $\begin{array}{l}\mathrm{Ap}^{\mathrm{r}}, \mathrm{Gm}^{\mathrm{r}} \text {, basic expression vector for Ptac and tac } \\
\text { RBS controlled expression }\end{array}$ & (Verhoef et al. 2010) \\
\hline $\mathrm{pJNTmcs}(\mathrm{t})$ & $\begin{array}{l}\mathrm{Ap}^{\mathrm{r}}, \mathrm{Gm}^{\mathrm{r}} \text {; basic expression vector containing the } \\
\text { salicylate-inducable promoter } n a g A a \text { and tac RBS }\end{array}$ & (Meijnen et al. 2008) \\
\hline pJNTxylAB_FGH & pJNTmes $(\mathrm{t})$ containing the $x y l A B_{-} F G H$ genes from $E$. coli $\mathrm{DH} 5 \alpha$ & (Meijnen et al. 2008) \\
\hline pBT'Tmcs & $\mathrm{Tc}^{\mathrm{r}}$; basic expression vector for Ptac and tac RBS controlled expression & This study \\
\hline pBT'Tpal & pBT'Tmes containing the pal gene from $R$. toruloides & This study \\
\hline pJQ200SK & P15A ori $s a c B$ RP4 Gmr(pBluescriptSK); suicide vector & (Quandt and Hynes 1993) \\
\hline pJQgcd::tetA_loxP & $\begin{array}{l}\mathrm{Gm}^{\mathrm{r}} \mathrm{Tc}^{\mathrm{r}}, \mathrm{pJQ} 200 \mathrm{SK} \text { containing a loxP-tetA-loxP } \\
\text { interrupted copy of the gcd gene }\end{array}$ & This study \\
\hline
\end{tabular}

${ }^{\mathrm{a}} \mathrm{Ap}^{\mathrm{r}}, \mathrm{Gm}^{\mathrm{r}}$ and $\mathrm{Tc}^{\mathrm{r}}$, ampicillin, gentamicin, and tetracycline resistance, respectively 
Upon reaching steady state at $D=0.1 \mathrm{~h}^{-1}$, the cultures were sampled again. For glucose-xylose mixtures, the $D$ was set at $0.1 \mathrm{~h}^{-1}$. The cultures were considered to be at steady state when no significant changes were measured in cell density, stirring speed, and $p$-hydroxybenzoate concentration after at least five volume changes at the corresponding $D$.

Analytical methods

Optical densities were measured at $600 \mathrm{~nm}\left(\mathrm{OD}_{600}\right)$ using an Ultrospec Cell Density Meter (GE Healthcare). An optical density of 1.0 corresponds to a cell dry weight (CDW) of $0.49 \mathrm{~g} \mathrm{l}^{-1}$. $p$-Hydroxybenzoate was analyzed by HPLC (Agilent 1100 system) using a Zorbax $3.5 \mu \mathrm{m}$ SB$\mathrm{C} 18$ column $(4.6 \times 50 \mathrm{~mm})$ and a diode-array detector set at $254 \mathrm{~nm}$. As the eluent, a linear gradient of acetonitrile in $\mathrm{KH}_{2} \mathrm{PO}_{4}$-buffer $(50 \mathrm{mM}, \mathrm{pH} 2,1 \%$ acetonitrile) was used, increasing from $0 \%$ to $25 \%$ in 4.9 min at a flow of

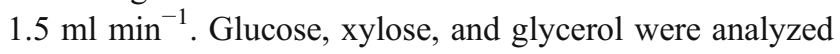
with a Dionex ICS3000 system as described previously (Meijnen et al. 2008; Verhoef et al. 2010).

\section{DNA techniques}

Genomic DNA was isolated using the DNeasy Blood \& Tissue kit (QIAGEN). PCR reactions were performed with Accuprime $P f x$ polymerase (Invitrogen) according to the manufacturer's instructions. Plasmid DNA was isolated with the QIAprep spin miniprep kit (QIAGEN). DNA concentrations were measured with the ND-1000 spectrophotometer (Nanodrop). Agarose-trapped DNA fragments were isolated with the QIAEXII gel extraction kit (QIAGEN). Plasmid DNA was introduced into electrocompetent cells using a Gene Pulser electroporation device (BioRad). DNA sequencing reactions were performed by Eurofins MWG Operon (Ebersberg, Germany).

\section{Construction of expression plasmids}

For constructing plasmid pBT'Tmcs $\left(\mathrm{Tc}^{\mathrm{r}}\right)$ the tac expression cassette and chloramphenicol $(\mathrm{Cm})$ marker of pJT'Tmcs (Verhoef et al. 2010) were amplified by PCR using primers 1 and 2 (Table 2). The resulting PCR product was digested with Kpn2I and XmaJI (restriction sites present in the amplified fragment) and consequently ligated in a Kpn2I$X b a I$ (compatible with XmaJI) digested pBBR1mcs vector, yielding $\mathrm{pBT}$ 'Tmcs $\left(\mathrm{Cm}^{\mathrm{r}}\right)$. The $\mathrm{Cm}$ marker was replaced by a tetracycline (Tc) marker, which was obtained by PCR using primers 3 and 4 (Table 2) on vector pTO1 (Kieboom and de Bont 2001) and cloned into the PagI and NcoI restriction sites of $\mathrm{pBT}$ 'Tmcs $\left(\mathrm{Cm}^{\mathrm{r}}\right)$, yielding $\mathrm{pBT}$ 'Tmcs $\left(\mathrm{Tc}^{\mathrm{r}}\right)$.

For constructing pBT'Tpal, the pal gene from pJTTpal (Verhoef et al. 2007) was obtained as a KpnI-NotI fragment and purified from agarose gel. The purified fragment was ligated into KpnI-NotI-digested pBT'Tmcs, yielding pBT'Tpal.

Expression vector pJNTxylAB_FGH was constructed by cloning $x y l A B$ and $x y l F G H$ in vector pJNTmcs (Meijnen et al. 2008). The genes were amplified by PCR using genomic DNA from E. coli $\mathrm{DH} 5 \alpha$ as the template and oligonucleotide primers 5-8 (Table 2). The resulting fragments were ligated into vector $\mathrm{pJNTmcs}$ using the restriction sites KpnI and NotI for $x y l A B$, and NheI and SfiI for $x y l F G H$. The resulting plasmid was designated pJNTxylAB_FGH.

Targeted gene disruption

The gcd knockout mutant of $P$. putida S12B2 (Verhoef et al. 2010) was constructed in analogy to the $g c d$ knockout mutant of wild-type P. putida S12 (Meijnen et al. 2008). The gene replacement plasmid for the $g c d$ gene, pJQgcd:: tetAloxP was constructed from the suicide vector $\mathrm{pJQ} 200 \mathrm{SK}$ (Quandt and Hynes 1993) based on the pJQged::Kana vector (Meijnen et al. 2008). The loxP-kanaR-loxP fragment, encoding kanamycin resistance in vector pJQged::Kana was replaced by the loxP-tetA-loxP fragment (Sauer and Henderson 1988; Sternberg and Hamilton 1981), coding for tetracycline resistance, using $\mathrm{XbaI}$.

\section{Results}

Construction of a xylose-utilizing $p$-hydroxybenzoateproducing strain

In order to establish $p$-hydroxybenzoate production from xylose, the optimized base strain for $p$-hydroxybenzoate production, P. putida S12B2 (Table 1; (Verhoef et al. 2010)), was engineered for xylose utilization. First, the $g c d$ gene encoding glucose dehydrogenase was disrupted in order to eliminate xylose oxidation, which makes xylose effectively unavailable for the xylose isomerase pathway (Meijnen et al. 2008). Subsequently, the $x y l A B \_F G H$ genes from E. coli $\mathrm{DH} 5 \alpha$ (encoding xylose isomerase, xylulokinase, and a high-affinity xylose transporter) were introduced. The resulting strain, P. putida S12xylB6, showed very slow growth on minimal medium with xylose as the sole carbon source, requiring 16 days to reach a cell dry weight $(\mathrm{CDW})$ of $0.69 \mathrm{~g} \mathrm{l}^{-1}$. This result was in agreement with previous findings, and evolutionary selection was performed to improve xylose utilization as described previously (Meijnen et al. 2008). After four transfers, $P$. putida S12xylB7 was obtained that exhibited a maximum growth rate of $0.34 \mathrm{~h}^{-1}$ on xylose and a biomass-tosubstrate yield $\left(Y_{\mathrm{xs}}\right)$ of $51 \mathrm{Cmol} \%$.

The phenylalanine/tyrosine ammonia lyase (Pal/Tal) expression plasmid $\mathrm{pBT}$ 'Tpal was introduced into $P$. putida 
Table 2 Oligonucleotide primers used in this study

\begin{tabular}{|c|c|c|}
\hline Primer & Sequence $^{\mathrm{a}}$ & Characteristics \\
\hline Primer 1 & TGACCAGCTGCGAAAGTG & Replicon of pJT'Tmes \\
\hline Primer 2 & $\begin{array}{l}\text { GCGA } \overline{\text { CGCGTGCGCACATTTC }} \\
\text { CC } \overline{\text { CGAAAAGTGC }}\end{array}$ & End of $\mathrm{Cm}^{\mathrm{R}}$ marker \\
\hline Primer 3 & $\begin{array}{l}\text { CGCGAATTCTCATGTTTGACA } \\
\text { GCTTATCATCG }\end{array}$ & Start of $\mathrm{Tc}^{\mathrm{R}}$ marker \\
\hline Primer 4 & $\begin{array}{l}\text { CGCGAATTCCCTCAGGTCG } \\
\text { AGGTGGCC }\end{array}$ & End of $\mathrm{Tc}^{\mathrm{R}}$ marker \\
\hline Primer 5 & $\begin{array}{l}\text { GCGGCGGGTACCATGGAGTT } \\
\text { CAATATGCAAGC }\end{array}$ & Start of $x y l A$ from E. coli, KpnI \\
\hline Primer 6 & $\begin{array}{l}\text { GCGGCGGCGGCCGCTTAC } \\
\text { GCCATTAATGGCAG }\end{array}$ & End of $x y l B$ from E. coli, Not $\mathrm{I}$ \\
\hline Primer 7 & $\begin{array}{l}\text { GCGGCGGCTAGCATGAAAAT } \\
\text { AAAGAACATTCTACTC }\end{array}$ & Start of $x y l F$ from $E$. coli, NheI \\
\hline Primer 8 & $\begin{array}{l}\text { GCGGCGGGCCTAGGCGGCCTC } \\
\text { AAGAACGGCGTTTGGTTGCGGA }\end{array}$ & End of $x y l H$ from E. coli, SfiI \\
\hline
\end{tabular}

${ }^{\mathrm{a}}$ The restriction sites used for cloning are underlined
Mixed-feed chemostats

S12xylB7 to establish $p$-hydroxybenzoate production, yielding strain P. putida S12pal_xylB7. Remarkably, upon introduction of pBT'Tpal, the maximum growth rate on xylose was reduced by a factor 3 compared to the plasmid-free strain, whereas the growth rate on glucose was not affected (results not shown). Still, $p$-hydroxybenzoate was efficiently produced from xylose in batch cultivations, at a product-to-substrate yield $\left(Y_{\mathrm{ps}}\right)$ of $12.4 \mathrm{Cmol} \%$ (Table 3). Another remarkable observation was the apparently improved $p$-hydroxybenzoate yield after evolutionary selection. Compared to the non-evolved strain P. putida S12palB6, the product-to-substrate yield of strain S12xyl_palB7 on glucose had increased from 14.2 to $17.4 \mathrm{Cmol} \%$. On glycerol, the product-to-substrate yield improved from 15.4 to $19.3 \mathrm{Cmol} \%$ (data not shown, respectively, Table 3 ).

Table 3 Product-to-substrate yields $\left(Y_{\mathrm{ps}}\right)$ of $P$. putida S12pal_xylB7 for various culture conditions and carbon sources

\begin{tabular}{llc}
\hline Cultivation & Carbon source & $Y_{\text {ps }}(\mathrm{Cmol} \%)$ \\
\hline Shake flask cultivation & Xylose & $12.4 \pm 0.51$ \\
Shake flask cultivation & Glucose & $17.5 \pm 0.07$ \\
Shake flask cultivation & Glycerol & $19.3 \pm 0.34$ \\
Chemostat cultivation $^{\mathrm{a}}$ & Xylose & $5.6 \pm 0.11$ \\
Chemostat cultivation $^{\mathrm{a}}$ & Glycerol & $8.3 \pm 0.22$ \\
Chemostat cultivation $^{\mathrm{a}}$ & Glycerol-xylose & $15.5 \pm 0.14^{\mathrm{b}}$ \\
Chemostat cultivation $^{\text {Chemostat cultivation }}$ & Glucose & $4.9 \pm 0.08$ \\
Chemostat cultivation $^{\text {Che }}$ & Glycerol & $8.1 \pm 0.06$ \\
Chemostat cultivation $^{\text {Glucose-xylose }}$ & $7.9 \pm 0.20^{\mathrm{b}}$ \\
\hline
\end{tabular}

Shake flask and chemostat cultivations were performed with $60 \mathrm{C} \mathrm{mM}$ total carbon, and chemostat cultivations were operated at a dilution rate of $0.1 \mathrm{~h}^{-1}$

${ }^{\text {a }}$ At a dilution rate of $0.05 \mathrm{~h}^{-1}$

${ }^{\mathrm{b}}$ Maximum value obtained
In previous work on the engineered xylose-utilizing $P$. putida strain S12xylAB2, a diauxic shift was observed in batch cultivations on mixtures of glucose and xylose, glucose being the preferred carbon source (Meijnen et al. 2008). Obviously, the occurrence of diauxy would invalidate the concept of co-feeding two substrates simultaneously to different pathways, in order to improve the availability of two key precursors. Therefore, a chemostat cultivation setup was selected for the mixed-substrate experiments, with glucose or glycerol as the limiting nutrient. Varying amounts of xylose were used $(0 \%$ to $75 \%$ of $60 \mathrm{mM}$ total carbon) to replace the primary carbon source.

Although the primary carbon source was completely metabolized as expected, residual xylose was observed in the chemostat effluent. The extent to which xylose was utilized was furthermore very different for glucose and glycerol (Fig. 2). On glycerol-xylose mixtures, the amount of xylose consumed correlated well with the amount of xylose in the feed. The residual xylose observed was in the same range as in chemostat cultivations with xylose as the sole carbon source (approximately $0.27 \mathrm{mM}$ ). However, with glucose as the primary carbon source, the residual xylose concentrations were much higher and, furthermore, increased more than proportionally to the relative xylose concentration in the feed. Clearly, the capacity to transport and/or utilize xylose was dependent on the type of primary carbon source as well as on the relative amount of the primary carbon source in the feed.

Production of $p$-hydroxybenzoate from mixtures of glucose and xylose

Chemostat cultivations with $P$. putida S12pal_xylB7 on glucose as single carbon source showed a product-tosubstrate yield of $4.9 \mathrm{Cmol} \%$, with a specific production 


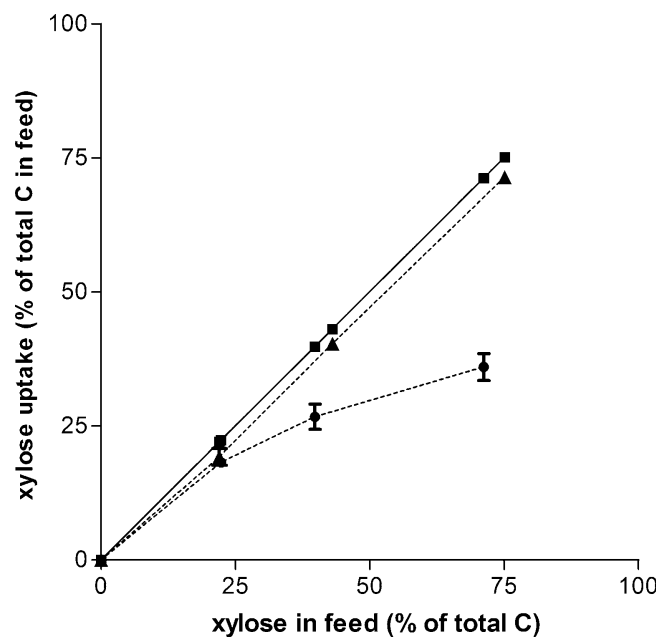

Fig. 2 Relative xylose uptake as a function of the xylose fraction in the feed. The solid line represents the theoretical maximum uptake of xylose; the dotted lines represent the actual uptake of xylose with glycerol as co-substrate (triangles) or glucose as co-substrate (circles). Data are the average from two independent cultivations; error bars represent the maximum deviation from the mean

rate of $8.0 \mu \mathrm{mol} \mathrm{C}(\mathrm{g} \mathrm{CDW})^{-1} \mathrm{~h}^{-1}$ (Fig. 3b). The biomass yield was $42.5 \mathrm{Cmol} \%$ (Fig. 3a). When xylose was co-fed with glucose, the biomass-to-substrate yield slightly increased with increasing xylose concentrations (Fig. 3a). This is in agreement with the higher biomass yield observed on xylose compared to glucose ( $Y_{\mathrm{xs}}$ of $47.7 \mathrm{vs}$. $42.5 \mathrm{Cmol} \%$, Fig. 3e, a). Moreover, the production of $p$ hydroxybenzoate improved significantly when xylose was co-fed with glucose. The product-to-substrate yield reached a maximum of $7.9 \mathrm{Cmol} \%$, and the specific production rate increased by a factor of 1.5 to $12.0 \mu \mathrm{mol} \mathrm{C}(\mathrm{g} \mathrm{CDW})^{-1} \mathrm{~h}^{-1}$ (Fig. 3b). These results clearly demonstrated that simultaneous utilization of glucose and xylose was beneficial for $p$ hydroxybenzoate production. Surprisingly, the amount of xylose that was co-fed did not significantly affect $Y_{\mathrm{ps}}$ and $q_{\mathrm{p}}$ within the range tested (Fig. 3b).

Production of $p$-hydroxybenzoate from mixtures of glycerol and xylose

In chemostats with glycerol as the sole carbon source, $P$. putida S12pal_xylB7 produced $p$-hydroxybenzoate at a yield of $8.5 \mathrm{Cmol} \%$. Notably, this represents a 1.7 -fold improvement over the product yield observed in glucosegrown cultures. Also in shake-flasks, an improved $p$ hydroxybenzoate yield on glycerol was observed (Table 3), although not as pronounced as in the chemostat cultivations. In addition to the product-to-substrate yield, also the biomass-to-substrate yield was improved 1.23-fold on glycerol (Fig. 3c). Because of the improved biomass yield, the effect of the primary substrate on the specific $p$ hydroxybenzoate production rate $q_{\mathrm{p}}$ was limited: the $q_{\mathrm{p}}$ on glycerol $\left(8.57 \mu \mathrm{mol} \mathrm{C}(\mathrm{g} \mathrm{CDW})^{-1} \mathrm{~h}^{-1}\right)$ was only slightly higher than on glucose $\left(7.96 \mu \mathrm{mol} \mathrm{C}(\mathrm{g} \mathrm{CDW})^{-1} \mathrm{~h}^{-1}\right)$.

The intrinsically improved $p$-hydroxybenzoate production from glycerol was further enhanced by xylose cofeeding. The product-to-substrate yield increased by a factor 1.9 to a maximum of $16.3 \mathrm{Cmol} \%$ (Fig. 3d). The $q_{\mathrm{p}}$ improved along with $Y_{\mathrm{ps}}$, to a maximum value of $18 \mu \mathrm{mol} \mathrm{C}(\mathrm{g} \mathrm{CDW})^{-1} \mathrm{~h}^{-1}$. As observed for the glucosegrown chemostats, the relative amount of xylose in the feed did not affect the efficiency of $p$-hydroxybenzoate production from glycerol-xylose mixtures (Fig. 3d).

Production of $p$-hydroxybenzoate from xylose as single carbon source

In order to verify that the improved $p$-hydroxybenzoate production was a result of the simultaneous utilization of xylose and glucose or glycerol, and not merely of xylose utilization alone, chemostat experiments were performed with $P$. putida S12pal_xylB7 on xylose as single carbon source. In agreement with the reduced growth rate observed in xylose-grown batch cultures after introducing the $\mathrm{pal} / \mathrm{tal}$ expression plasmid, P. putida S12pal_xylB7 washed out above a dilution rate of $0.05 \mathrm{~h}^{-1}$. Therefore, chemostats on xylose were operated at a dilution rate of $0.05 \mathrm{~h}^{-1}$. Also chemostats with a mixed glycerol-xylose feed, or glycerol as the sole carbon source, were operated at this dilution rate, as control for growth-rate effects on $p$-hydroxybenzoate production.

Biomass and product yields were not significantly different in chemostats operated at $D=0.05 \mathrm{~h}^{-1}$ and $D=$ $0.1 \mathrm{~h}^{-1}$ (Fig. 3c-f), whereas the specific production and substrate uptake rates were proportional to the dilution rate as expected (Fig. 3e-f). On xylose as a single carbon source, the product-to-substrate yield $(5.6 \mathrm{Cmol} \%)$ was 1.5 fold lower than on glycerol alone (Table 3), which result is in agreement with the product yields observed in shake flask cultures (Table 3). This demonstrates that the improved $p$-hydroxybenzoate production is indeed a result from co-feeding two carbon sources. Furthermore, as the product yield on mixtures of xylose and glycerol or glucose consistently exceeded the product yield on either individual carbon source (Table 3), it was concluded that co-feeding xylose to glucose or glycerol has a beneficial effect over feeding individual carbon sources for $p$-hydroxybenzoate production.

\section{Discussion}

A mixed-substrate feeding strategy was devised to improve aromatics production by engineered $P$. putida S12. The approach was based on the assumption that the precursors 
Fig. 3 Growth and $p$-hydroxybenzoate production of $P$. putida S12pal_xylB7 in chemostat cultivations on various mixtures of carbon sources at various dilution rates $(D)$. The biomassto-substrate yield $\left(Y_{\mathrm{xs}}\right)$, productto-substrate yield $\left(Y_{\mathrm{ps}}\right)$, and product-to-biomass yield $\left(Y_{\mathrm{px}}\right)$ expressed as in $\mathrm{Cmol}$ biomass or product per $\mathrm{Cmol}$ substrate or biomass. The specific carbon uptake rate $\left(q_{\mathrm{s}}\right)$ and specific $p$-hydroxybenzoate production rate $\left(q_{\mathrm{p}}\right)$ were given in $\mathrm{C} \mu \mathrm{mol}$ substrate or product per gram cell dry weight (CDW) per hour. All calculated figures were corrected for the consumed amount of substrate glucose and xylose mixtures at a D of $0.1 \mathrm{~h}^{-1}$

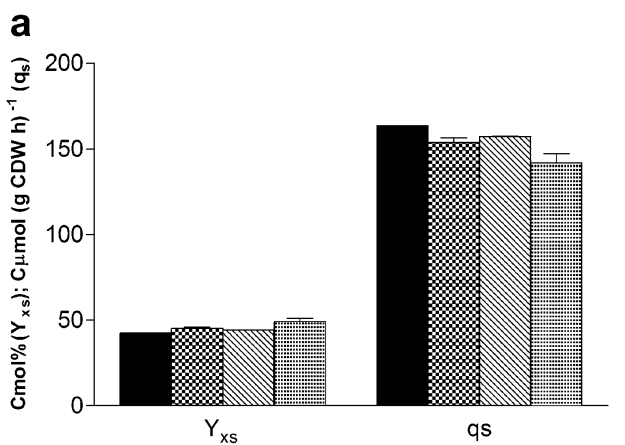

b

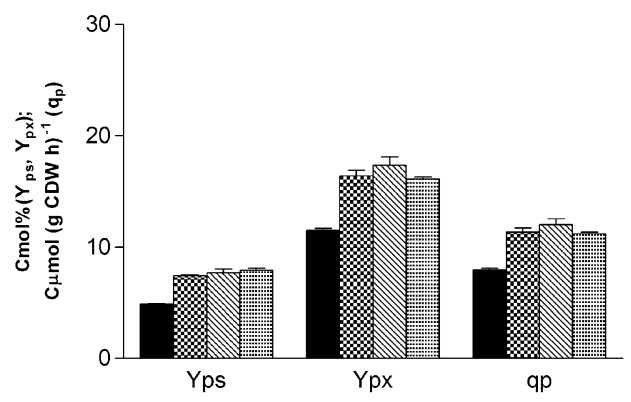

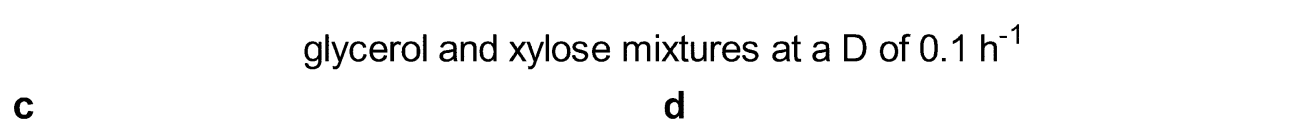
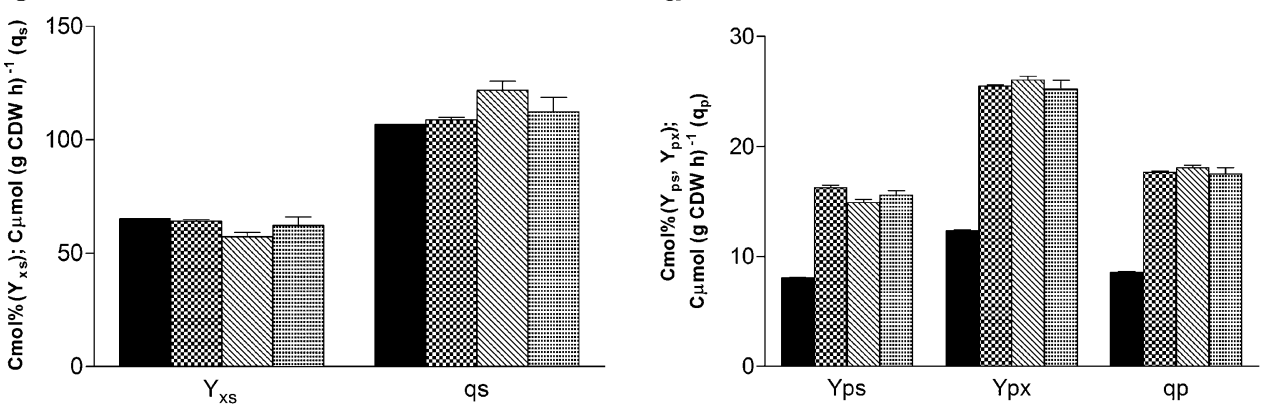

glycerol and xylose mixtures at a $D$ of $0.05 \mathrm{~h}^{-1}$

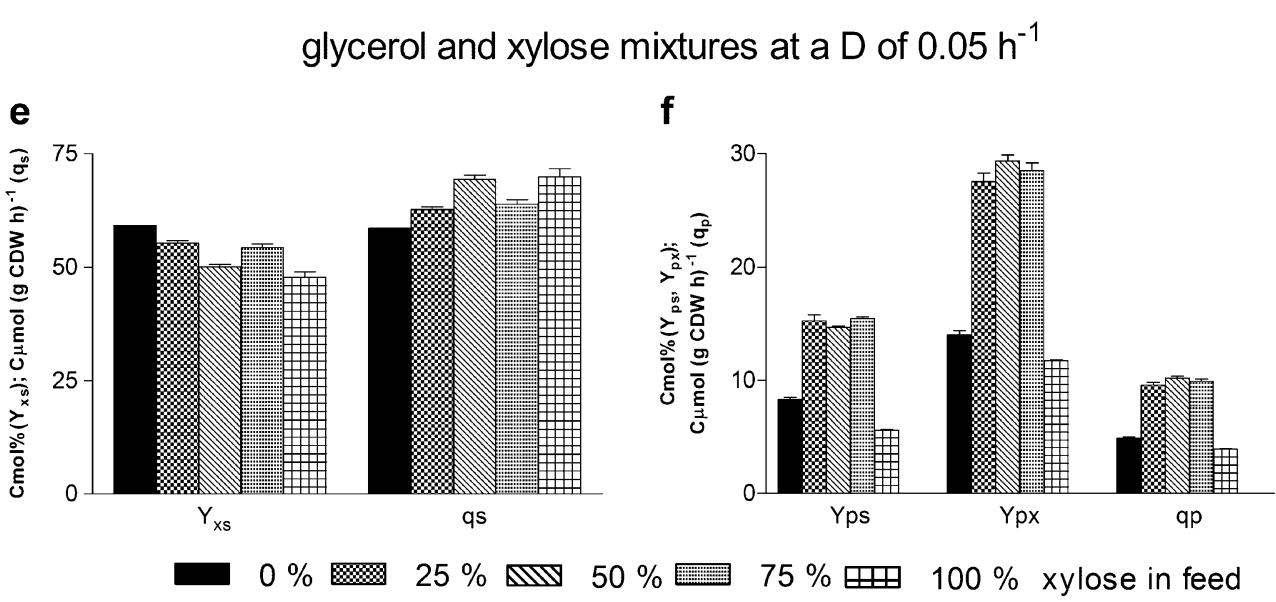

E4P and PEP were limiting factors for aromatic biosynthesis and that their availability could be improved by stimulating the PP pathway fluxes through pentose cofeeding. A $p$-hydroxybenzoate producing strain, P. putida $\mathrm{S} 12 \mathrm{palB} 2$, was selected as an aromatics-producing model system. As this strain does not have the natural ability to utilize pentoses, a xylose isomerase pathway was introduced, and the initially low growth rate on xylose was improved via an evolutionary selection procedure.

Surprisingly, tenfold less transfers were required to achieve growth characteristics similar to those of the previously evolved xylose-utilizing strain $P$. putida S12xylAB2 (Meijnen et al. 2008). In part, this can be attributed to the targeted disruption of $g c d$, since more than ten transfers had been required for strain S12xylAB2 to acquire a $g c d$ negative phenotype. In addition to the xylose utilization efficiency, also the $p$-hydroxybenzoate yield was improved after the evolutionary selection. This phenomenon may be explained by the increased PP pathway activity associated with the improved xylose utilization phenotype, leading to an intrinsically improved E4P and PEP availability, independently from xylose co-feeding.

As anticipated, xylose co-feeding considerably improved the $p$-hydroxybenzoate yield. The increased product yield was observed with both glucose and glycerol as the primary substrate and was shown not to be caused by xylose consumption per se. Remarkably, both the product and biomass yield on glycerol were consistently higher com- 
pared to glucose, either with or without xylose co-feeding. This may be attributed to regulatory effects (e.g., carbon catabolite repression) but could also indicate that PEP availability is more critical for efficient $p$-hydroxybenzoate production than E4P availability. If it is assumed that pyruvate dikinase (PEP synthase) is active only under gluconeogenic conditions (Sauer and Eikmanns 2005), twice the amount of GAP (and, thus, PEP) can be obtained from glycerol compared to glucose, which is metabolized via the Entner-Doudoroff pathway in pseudomonads (del Castillo et al. 2007; Fuhrer et al. 2005). In addition, the glycerol-associated yield improvement appears to be connected to the evolutionary selection, since no such effect has been observed with the parent strains of $P$. putida S12pal_xylB7 (Verhoef et al. 2010). Presumably, the increased PP pathway activity associated with the efficient xylose-utilizing phenotype may allow for a more efficient equilibration of PEP and E4P levels, resulting in more efficient $p$-hydroxybenzoate production. It should be noted that the applied proportion of xylose in the feed showed little effect within the range tested, whether the primary substrate was glucose or glycerol. Apparently, the $p$ hydroxybenzoate production is not very sensitive to variations in relative xylose concentrations above a certain threshold value.

Unexpectedly, the capacity to transport and/or utilize xylose appeared to be dependent on the primary carbon source. With glycerol, a low concentration of residual xylose was observed that is presumably close to the $K_{\mathrm{m}}$ of the-yet unidentified-xylose transporter in P. putida S12pal_xylB7. With glucose as the primary substrate, however, the residual xylose concentrations were higher and furthermore increased more than proportionally with increasing amounts of xylose in the feed. Although this phenomenon is still subject to further study, it may be hypothesized that xylose transport in P. putida S12pal_xylB7 is PEP dependent. This would be consistent with the observed increase in residual xylose concentrations with decreasing glucose feed (an already relatively inefficient source of PEP), the relative independency between residual xylose concentration and glycerol feed (a good source of PEP), and the decreased growth rate on xylose when $\mathrm{Pal} / \mathrm{Tal}$ was introduced (drain on PEP for $p$-hydroxybenzoate production). In that case, replacing any PEP-dependent transport systems would be an obvious target for further strain improvement. The GAP/PEP availability may furthermore be improved by constructing an ED-negative, glycolytic P. putida S12 strain. The contribution of the (ATP-driven) E. coli xylose transporter XylFGH to xylose import was presumably limited as observed previously (Meijnen et al. 2008).

We have demonstrated that $p$-hydroxybenzoate production in P. putida can be considerably improved by cofeeding different carbon sources that are metabolized via different, (partly artificial) pathways. Thus, the availability of the key aromatics precursors, PEP and E4P, is improved. In addition to $p$-hydroxybenzoate, the production of other aromatic compounds derived from aromatic amino acids may be stimulated via this strategy. Moreover, lignocellulosic hydrolysate, the expected major feedstock for future production of biobased fuels and chemicals (Himmel and Bayer 2009; Kumar et al. 2008; Lange 2007), seems to be ideally suited for aromatics production since glucose and xylose are the predominant constituents. Also the improved production on glycerol presents an additional possibility to deploy a cheap and abundant waste substrate for biocatalytic production of (fine) chemicals.

Acknowledgements The authors thank Karin Nijkamp for constructing the basic expression plasmids. This project is financially supported by the Netherlands Ministry of Economic Affairs and the BBasic partner organizations (http://www.b-basic.nl) through B-Basic, a public-private NWO-ACTS programme $(\mathrm{ACTS}=$ Advanced $\mathrm{Chemical}$ Technologies for Sustainability). This project was co-financed by the Kluyver Centre for Genomics of Industrial Fermentation, which is part of the Netherlands Genomics Initiative/Netherlands Organization for Scientific Research.

Open Access This article is distributed under the terms of the Creative Commons Attribution Noncommercial License which permits any noncommercial use, distribution, and reproduction in any medium, provided the original author(s) and source are credited.

\section{References}

de Bont JA (1998) Solvent-tolerant bacteria in biocatalysis. Trends Biotechnol 16:493-499

del Castillo T, Ramos JL, Rodriguez-Herva JJ, Fuhrer T, Sauer U, Duque E (2007) Convergent peripheral pathways catalyze initial glucose catabolism in Pseudomonas putida: genomic and flux analysis. J Bacteriol 189:5142-5152

Fuhrer T, Fischer E, Sauer U (2005) Experimental identification and quantification of glucose metabolism in seven bacterial species. $\mathrm{J}$ Bacteriol 187:1581-1590

Gonzalez R, Tao H, Shanmugam KT, York SW, Ingram LO (2002) Global gene expression differences associated with changes in glycolytic flux and growth rate in Escherichia coli during the fermentation of glucose and xylose. Biotechnol Prog 18:6-20

Hartmans S, van der Werf MJ, de Bont JA (1990) Bacterial degradation of styrene involving a novel flavin adenine dinucleotide-dependent styrene monooxygenase. Appl Environ Microbiol 56:1347-1351

Heipieper HJ, Neumann G, Cornelissen S, Meinhardt F (2007) Solvent-tolerant bacteria for biotransformations in two-phase fermentation systems. Appl Microbiol Biotechnol 74:961-973

Himmel ME, Bayer EA (2009) Lignocellulose conversion to biofuels: current challenges, global perspectives. Curr Opin Biotechnol 20:316-317

Kieboom J, de Bont JAM (2001) Identification and molecular characterization of an efflux system involved in Pseudomonas putida S12 multidrug resistance. Microbiology 147:43-51

Kumar R, Singh S, Singh OV (2008) Bioconversion of lignocellulosic biomass: biochemical and molecular perspectives. J Ind Microbiol Biotechnol 35:377-391 
Lange JP (2007) Lignocellulose conversion; an introduction to chemistry, process and economics. Biofuels, Bioprod Biorefin $1: 39-48$

Martinez K, de Anda R, Hernandez G, Escalante A, Gosset G, Ramirez OT, Bolivar FG (2008) Coutilization of glucose and glycerol enhances the production of aromatic compounds in an Escherichia coli strain lacking the phosphoenolpyruvate: carbohydrate phosphotransferase system. Microb Cell Fact 7:1

Meijnen JP, de Winde JH, Ruijssenaars HJ (2008) Engineering Pseudomonas putida S12 for efficient utilization of D-xylose and L-arabinose. Appl Environ Microbiol 74:5031-5037

Nijkamp K, van Luijk N, de Bont JA, Wery J (2005) The solvent-tolerant Pseudomonas putida $\mathrm{S} 12$ as host for the production of cinnamic acid from glucose. Appl Microbiol Biotechnol 69:170-177

Nijkamp K, Westerhof RGM, Ballerstedt H, de Bont JAM, Wery J (2007) Optimization of the solvent-tolerant Pseudomonas putida S12 as host for the production of $p$-coumarate from glucose. Appl Microbiol Biotechnol 74:617-624

Quandt J, Hynes MF (1993) Versatile suicide vectors which allow direct selection for gene replacement in gram-negative bacteria. Gene 127:15-21

Sambrook J, Fritsch EF, Maniatis T (1982) Molecular cloning. A laboratory manual. Cold Spring Harbor Laboratory Press, Cold Spring Harbor

Sauer U, Eikmanns BJ (2005) The PEP-pyruvate-oxaloacetate node as the switch point for carbon flux distribution in bacteria. FEMS Microbiol Rev 29:765-794
Sauer B, Henderson N (1988) Site-specific DNA recombination in mammalian cells by the Cre recombinase of bacteriophage P1. Proc Natl Acad Sci USA 85:5166-5170

Sternberg N, Hamilton D (1981) Bacteriophage P1 site-specific recombination. I. Recombination between loxP sites. J Mol Biol 150:467-486

Verhoef S, Ruijssenaars HJ, de Bont JA, Wery J (2007) Bioproduction of $p$-hydroxybenzoate from renewable feedstock by solventtolerant Pseudomonas putida S12. J Biotechnol 132:49-56

Verhoef S, Wierckx N, Westerhof RG, de Winde JH, Ruijssenaars HJ (2009) Bioproduction of $p$-hydroxystyrene from glucose by the solvent-tolerant bacterium Pseudomonas putida S12 in a twophase water-decanol fermentation. Appl Environ Microbiol 75:931-936

Verhoef S, Ballerstedt H, Volkers RJ, de Winde JH, Ruijssenaars HJ (2010) Comparative transcriptomics and proteomics of $p$-hydroxybenzoate producing Pseudomonas putida S12: novel responses and implications for strain improvement. Appl Microbiol Biotechnol 87:679-690

Wierckx NJP, Ballerstedt H, de Bont JAM, Wery J (2005) Engineering of solvent-tolerant Pseudomonas putida S12 for bioproduction of phenol from glucose. Appl Environ Microbiol 71:8221-8227

Wierckx N, Ruijssenaars HJ, de Winde JH, Schmid A, Blank LM (2009) Metabolic flux analysis of a phenol producing mutant of Pseudomonas putida S12: verification and complementation of hypotheses derived from transcriptomics. J Biotechnol 143:124 129 